\title{
Onboard Secondary Power Supply Automatic Test Hardware Design and Implementation
}

\author{
Ning Wang ${ }^{1, a}$ and Chong zhao ${ }^{2, b}$ \\ 1,2Xijing University, Shaanxi Xi'an, 710123, China \\ a 2571449451@qq.com, ${ }^{\text {b } 36784625 @ q q . c o m, ~}$
}

Keywords: Hardware; Secondary power; MSP430; Communication; Load

\begin{abstract}
In order to achieve test accuracy, the hardware component of the secondary power test system mainly adopts the more mature products in the market. Therefore, as the design of products, this paper describes the design scheme of load cases, including microprocessor MSP430 minimum system design, communication interface circuit design, circuit design load and multiplex called the design of the road.
\end{abstract}

\section{Introduce}

Along with the rapid development of science and technology, all kinds of such as satellite, missile, rocket and airplane aircraft, involving growing and gradually tend to be more complicated electronic equipment, also increase accordingly to the requirement of the power supply system. The aircraft power supply system for a single set of voltage power supply, but by the spacecraft of various modules for different power supply voltage, in order to meet the demand of different devices, the secondary power supply arises at the historic moment. Is the main purpose of the secondary power supply converts aircraft power supply system to provide electrical energy to another or multiple forms of energy, to meet the demand of different equipment power supply device. Because the secondary power supply can meet the demand of all kinds of modules in the aircraft, its function in the power supply system is very important.

\section{The Design of the MSP430 Core System}

As one of TI Company has an electronic product, MSP430 series MCU ultra-low power consumption characteristics of high integration, with its own advantage industry wide recognition and application. The physical image of the MSP430 chip is shown in Fig.1.

Ultra-low power consumption; Efficient development environment; Strong data processing and operational capability; A stable working environment; High-performance analog technology; In system integration, the MSP430 single-chip microcomputer integrates multiple modules based on different products. These modules involve timers, analog comparators, hardware multiplier, 10/12/14/16 ADC, DMA controllers, 10K RAM and SVS, multifunctional serial interfaces (SPI/IIC/UART). There are also rich interruptions.

\section{MSP430F552x \\ MCUs \\ 4 is TEXAS}

Figure 1 physical photos of MSP430

In addition to the features of the single chip, this series of singlechip sets features in several aspects:

\section{Power Circuit}

The main manifestation of the low power consumption of the MSP430 single-chip microcomputer is that the power supply is low, mostly in the range of $1.8 \sim 3.6$ volts. Considering 
the testing system of digital oscilloscope and other equipment used $220 \mathrm{v}$ alternating current (ac), to facilitate the actual operation, the load cases of the power supply adopts the design pressure, turn $220 \mathrm{v}$ alternating current $(\mathrm{ac})$ for $5 \mathrm{v}$ dc. This is the ability to meet the power requirements of a lot of relays, and to get a dc power supply for a single chip.

Since the MSP430 single-chip machine has a voltage of $3.3 \mathrm{~V}$, and the voltage of the transformer output is $5 \mathrm{~V}$, the voltage adjustment chip AMS1117 is used for the voltage adjustment. AMS1117 internal integration, overheating protection and current limiting circuit suitable for laptop computers, battery chargers, SCSI II active terminals, mobile phones, batteries, power supply system and SMPS post voltage regulator, etc.

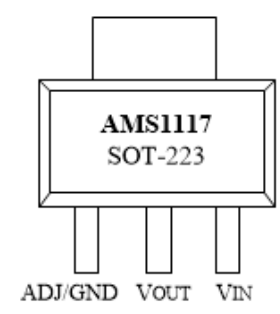

Figure 2 AMS117's pins

The pins of the AMS1117 are shown in Fig. 2. The number 1 is fixed, adjustable voltage output/ground, number 2 is the output voltage, and the number 3 is the input voltage.

The MSP430 power supply system circuit diagram is shown in Fig. 3. Since only the AMS1117 chip fixed version of two input and output capacitor and load, the peripheral circuit is simple, it adopted the fixed version 3.3 V output, and its voltage control pipe is composed of a PNP driver of NPN transistors. The power supply of the load box is $220 \mathrm{~V}$ alternating current, converted to $5 \mathrm{~V}$ direct current by transformer, access to JP1 interface, and then Switch Switch by power Switch. From the input 5V voltage, the stabilized pressure chip AMS1117 provides a fixed $3.3 \mathrm{~V}$ output voltage. The capacitance of the input and output terminals of the chip, the circuit is filtered and the noise is reduced. Through further filtering, the VDD side picks up one of the MSP430's pins, which is powered by the digital portion; the magnetic beads inhibit the high frequency noise and peak interference of the signal line, the power line, and the end of the socket from the end of the line, and the power supply of the analog parts.

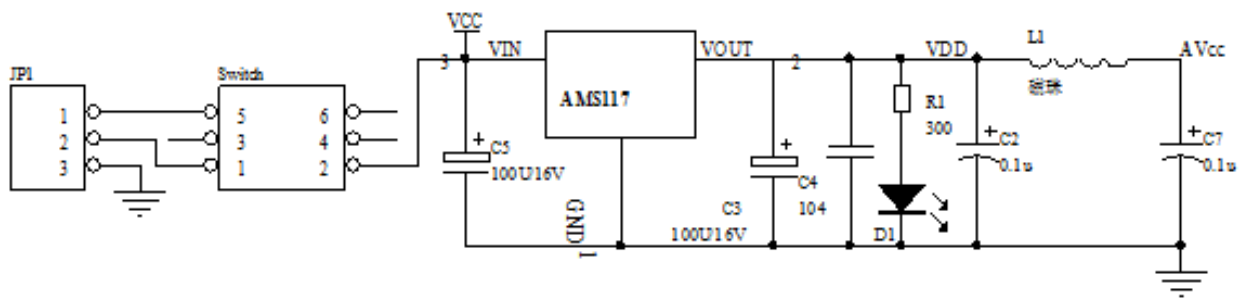

Figure 3 MSP430 power supply system circuit diagram

\section{Reset Circuit}

According to the functional requirements to test system, the periphery of the single chip microcomputer circuit design is relatively simple, in addition to the above tells the MSP430 power supply circuit, clocking circuit and reset circuit two parts. The MSP430 hardware reposition includes both electrical and manual reset. MSP430 reposition drawing RST low level is valid. 


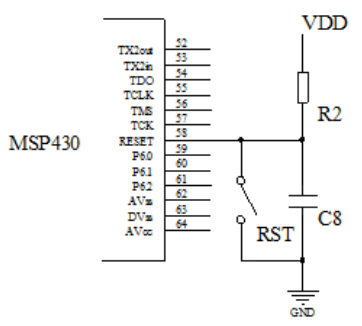

Figure 4 MSP430 reposition circuit diagram

The MSP430 reposition circuit diagram is shown in Fig. 4. When the MSP430 is energized, the capacitor is in a low level state. As the process of charging is carried out, the electric level of the capacitance is raised gradually, and it is maintained, and the electric reset is completed automatically. Press the RST reset button, the RET port voltage is pulled low and the manual reset is done.

\section{Clock Circuit}

Because the clock system exists, the individual modules of the single chip can work with the rhythm of the clock signal. The MSP430 contains low speed oscillator for low power consumption and $32768 \mathrm{~Hz}$ vibration. In addition, when higher working voltages are required on some occasions, the system requires a higher power. A single chip can be used by an external high-speed crystal oscillator $(450 \mathrm{kHz} \sim 8 \mathrm{MHz})$ to make it work in high frequency mode.

The clock circuit diagram for MSP430 is shown in Fig. 5. The quartz crystal Y2 consists of two pins connected by XIN and XOUT and two $15 \mathrm{pF}$ capacitors, making the external low speed clock of the MSP430. The quartz crystal Y1 consists of an external high speed clock of the MSP430 by using the two pins, XT2OUT and XT2IN, and two $15 \mathrm{pF}$ capacitors.

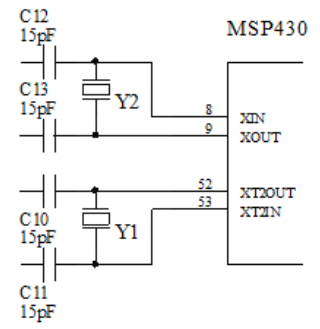

Figure 5. MSP430 clock circuit diagram

\section{Communication Interface Circuit Design}

Because RS232 has wide application range, cheap, and easy in programming, its performance can meet the demand of system each module of communication, so this design adopts RS232 to realize the upper machine and lower machine communication. But in a computer USB serial port to support multiple peripherals, application is more convenient, thus has been adopted in the second chapter introduces the USB/RS232 converter, namely RS232 data transmission via USB bus, namely the PC application software is still in view of the RS232 serial port programming, peripherals is with RS232 communication channel for the data, but the physical connection between from PC to peripheral is USB bus, USB data communication and data format.

Since the RS232 number is using negative logic, a level conversion chip is required for a single chip connection. In order to achieve the communication of MSP430 with the above machine, special serial chip is required to carry out the data transfer between the two, and the chip MAX3222 is selected for this. This is a chip for the RS232 interface circuit, which is used exclusively for computer and single-chip computers, and supports maximum transmission speed of $1 \mathrm{Mbps}$. The $220 \mathrm{~V}$ alternating current (220V alternating current) of the load box power supply is referred to as + $5 \mathrm{~V}$ direct current, which meets the demand of the chip. 
With the RS232 protocol output level, MAX3222 ensures the data transfer rate of $120 \mathrm{kbps}$, with two receivers and two drives. The MAX3222 $1 \mathrm{mu}$ A shut off mode feature can reduce the power consumption effectively and prolong the battery life of the portable system. In the closed mode, the receiver remains active while allowing only one mu A working current to monitor external equipment.

\section{Load Circuit Design}

According to design requirements, for each power supply under test, all the way to provide light, 50\% load and $85 \%$ load and full load status, relative to the light condition is relatively simple, not loaded. If using a single load state corresponding to a single road load method, with the improvement of resistance power, the design of the corresponding cost will also increase, in addition, the individual resistance of higher power problems such as difficult to buy. Therefore, in order to save the design cost, the combination of the parameter requirements, the method of load stacking is used to solve the above problem. The rest will be explained in a different way because of the similar power supply. The following will be described in a certain way.

The load circuit diagram is shown in Fig.6. Provides a sub board work load circuit schematic diagram, among them, and said the relay control circuit on and off,,,, for three road load resistance, said to be IN power input, the OUT said the output of the power supply under test.

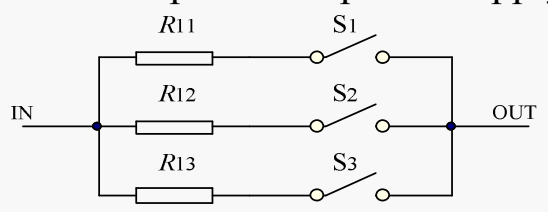

Figure 6. Load circuit work schematic

\section{More than Ways to Select the Working Principle}

Between the main board and the corresponding 11 circuit, the connection between the power source and the power circuit is connected by the connector $\mathrm{J} 1 \sim \mathrm{J} 4$. There is a one-to-one correspondence between the MSP430 and the 11-pending circuit, which is the single chip MSP430, which corresponds to the circuit of the power supply. So as each relay logic control part of the relay on the wired way, exist in "exclusive" logical way, guarantee from single-chip computer MSP430 to called the road between the reliability and security. In addition, to combine the program control of the upper and lower machine, the command is sent to enable each energy to be selected only for the second power supply.

\section{References}

[1] Effrey Travis, Jim Kring. LabVIEW for Everyone: Graphical Programming Made Easy and Fun,Third Edition[M].Person Education,Inc,2007.

[2] M. Malesich. Advances in DoD's ATS framework. Aerospace and Electronic Systems Magazine. 2008,23(2): 11 16.

[3] C.Schwarz, C.Pothier. Commercial ATS program management.Aerospace and Electronic Systems Magazine. 2002, 17(12): 3 6.

[4] D.R.Carey. Tobyhanna army depot Automated Test System modernization.Aerospace and Electronic Systems Magazine. 2011, 26(9): 22 26. 